\title{
Relationship Between c-Jun N-terminal Kinase and Depression
}

\author{
Hongpeng $\mathrm{Ma}^{1}$ \\ ${ }^{1}$ Shandong University, School of Medicine, 250100 Jinan, China
}

\begin{abstract}
Depression is one of the most common emotional disorders. The cause of depression is still not clear. C-Jun N-terminal kinase (JNK) is one number of mitogen activated protein kinase (MAPK) family, which is closely related to the occurrence of many diseases. At present, it is believed that JNK plays an important role in the parthenogenesis of depression, but the specific mechanism is not clear. This review will focus on the possible mechanism of JNK protein and JNK signaling pathway affecting the parthenogenesis of depression.
\end{abstract}

\section{Introduction}

Depression is the main type of mood disorder, which is characterized by its clinical feature of significant and long-lasting low state of mind. The typical clinical manifestations of depression can be listed as follows: low state of mind being out of proportion to the situation, mental retardation and hypo volition, sleep disorders, anorexia and other physical symptoms. Patients with depression often have obvious anxiety symptoms, which may lead to suicide attempts or behaviors. Severe patients even have psychotic symptoms such as hallucination and delusion. The incidence is around $15 \%$ worldwide.[1] It also has a high disability rate and mortality rate, which seriously endangers human health. With the development of society and the acceleration of work and life rhythm, the incidence of depression is increasing.[2] The cause of depression is not clear yet. It is generally believed that it is the result of the interaction of environment, society and individual.

In recent years, it is believed that C-Jun N-terminal kinase (JNK) plays an important role in causing depression. JNK is involved in several processes including cell proliferation, differentiation and apoptosis. JNK protein is encoded by three genes, which are JNK1, JNK2 and JNK3. Normally, JNK1 and JNK2 are widely expressed in the whole body, while JNK3 is only expressed in the heart, brain and testis.[3] Under the action of cytokines, inflammatory response, oxidative stress and other extracellular stimuli[4], MAP kinase kinase (MKK) such as MKK4[5] is activated by phosphorylation of MAP kinase kinase kinase (MKKK), so as to activate its downstream factor JNK. In the process of JNK activation, MAPK family pathway cross talk and signal integration[6] may exist. When JNK activated by upstream factors, it will act on the amino terminal of transcription factor c-Jun, making its terminal ser-63 and Ser-73 phosphorylated, so as to activate c-Jun transcription factor.[7] Different subtypes of JNK protein kinases can selectively interact with different transcription factors to regulate various cell vital movements.[8]

In a word, understanding the role of JNK signaling pathway in depression is very important to comprehend the parthenogenesis of depression and develop new targeted therapies. In this review, we will first introduce JNK signaling pathway and the factors that affect its activity. Then we will mainly focus on the possible mechanism of JNK involved in the course of depression. In addition, we will discuss the possibility of JNK as a target medicine for depression treatment.

\section{FACTORS AFFECTING JNK}

A variety of positive and negative regulatory factors act on the upstream of $\mathrm{JNK}$, adjusting the activation and expression of JNK.

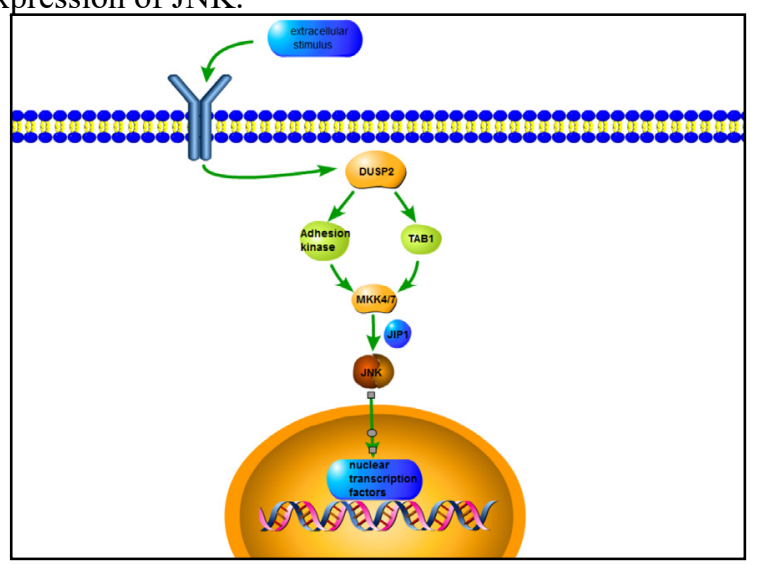

Fig. 1. The process of activating JNK by DUSP2 and JIP1. | DUSP2 can activate JNK signaling pathway through two downstream factors: adhesion kinase and TAB1. At the same time, JIP1 plays a role in JNK phosphorylation by MKK.

The JNK pathway has a wide range of regulatory factors, belonging to a variety of biological or nonbiological stress events, such as infection, inflammation, oxidative stress, DNA damage, osmotic stress or

\footnotetext{
${ }^{*}$ Corresponding author: mah1001@foxmail.com
} 
cytoskeleton changes. Here are just some examples of JNK regulatory factors. In the phosphorylation cascade activation pathway, Specific Ser / Thr phosphatase may regulate the amplitude and time of JNK signal activation through its role in multiple levels of JNK pathway.[9] Dual-specificity phosphatase (DUSP2) can not only dephosphorylate phosphotyrosine and phosphoserine / threonine residues in substrate proteins, but also can regulate the activity of JNK pathway by regulating adhesion kinase[10] and TAB1[11] activation. As an upstream component of JNK pathway, JNK-interacting protein 1 (JIP1) switches between anterograde and retrograde complexes to regulate axonal transport[12], JIP can also participate in JNK pathway regulation of kinesin-1-dependent intracellular transport.[13](Figure.1)

At the same time, JNK pathway is also affected by feedback. The activated JNK can directly act on the phosphorylated mixed lineage kinase 3 (MLK3), and then realize the positive feedback to its loop.[14] Colistin induced autophagy and apoptosis are involved in the positive feedback regulation of JNK-Bcl2-Bax signaling pathway.[15] Drugs related to cell cycle regulators act on the synergetic and antagonistic feedback loop formed by JNK / pJNK and p53 / p53.[16] Via WSSV-IE1, white spot syndrome virus (WSSV) combines with JNK and enhances JNK activation through self-phosphorylation, then establishes a positive feedback loop of JNK mediated by virus gene.[17]

\section{JNK AND DEPRESSION}

\subsection{JNK and Inflammation}

The activation of JNK pathway leads to depression related inflammatory response, and many inflammatory factors participate in this process. Moreover, long term exposure to glucocorticoids can cause mental disorders and insulin resistance, the increasing expression of JNK and HPA axis disorders play an important role in this process.[18] Cortisol and sex hormone levels are involved in the regulation of depression by HPA axis.

\subsubsection{JNK and inflammatory factors}

Stimulating factors like LPS can increase the expression of proinflammatory cytokines, the activation of JNK and the phosphorylation of glucocorticoid receptor (GR) in the brain, and then cause depression like behavior. The over activation of $\mathrm{JNK}$ is involved in the expression of pro-inflammatory cytokines, the regulation of GR phosphorylation and the occurrence of neuritic depression by regulating the activities of $\mathrm{Hb}$, amyg and mPFC.[19](Figure.2)

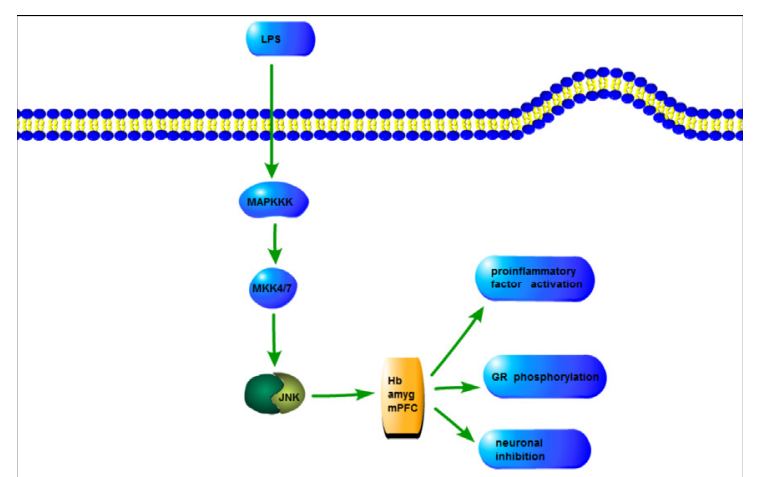

Fig. 2. Inflammatory factors in the activation of JNK. | Under the stimulation of LPS and other factors, JNK cascade signaling pathway was activated. JNK acts on the downstream factors such as $\mathrm{Hb}$, which leads to the activation of proinflammatory factors, GR phosphorylation and neuron inhibition.

There are reasons to believe that JNK signaling pathway induces secondary reactions by increasing the expression of TNF - $\alpha$ and other inflammatory cytokines, and plays a role in the upstream and downstream of the signal, causing depression related neuroinflammatory reactions.[20] It can also phosphorylate interferon regulator factor (IRF) and induce transcription of chemokines and cytokines in innate immunity.[21] Blocking JNK pathway could apparently diminish C1qtumor necrosis factor-related protein-3(CTPR3) and lead to cognitive impairment, inflammatory response and neuron death.[22] In depression regulated by CTRP3, JNK signaling pathway has an important role.

\subsubsection{JNK and regulation axis of endocrine system}

In bipolar disorder patients, JNK's intracellular signal transduction changes, and then regulates the pathological processes such as hypothalamic pituitary adrenal (HPA) axis hyperactivity, glucocorticoid insensitivity, and change of inflammatory mediators. [23]In the study of other cognitive disorders, JNK activation was found to be involved in the pathway between insulin resistance and cognitive impairment.[24] The experiment results showed that JNK could increase the inflammatory response and insulin resistance.[25] In addition, the continuous activation of JNK in pancreatic $\beta$ cells may lead to the decrease of insulin secretion and the loss of $\beta$ cell function, which is also an unfavourable factor of depression.[26] Cytokines such as IL-1 are involved in this process.[27] Adrenalectomy (ADX) increases the content of $\mathrm{pJNK} / \mathrm{JNK}$ and insulin negative regulatory factors in hypothalamus, resulting in the related hypophagia and responses in the hypothalamus.[28]

In addition, JNK also plays a role in the pituitary thyroid axis.It was found that the presence of JNK is a necessary condition to maintain the expression of TSH $\beta$, which also participates in insulin resistance and inflammation.[29] It is reasonable to believe that the pituitary thyroid axis disorder mediated by JNK change is also related to depression. 


\subsection{JNK, oxidative stress and cell death}

\subsubsection{JNK and oxidative stress}

Stress is one of the important factors of depression susceptibility. Considered as a stress-activated protein kinase(SAPKs), JNK can be activated by cell stress and TNF- $\alpha .[30]$ Stress-related experiments show that the activation of JNK phosphorylation pathway plays an important role in the process of depression.[31] The increased level of JNK affects the resistance of mice to social defeat stress (SDS).[32]

Reactive oxygen species(ROS) can trigger JNK cascade reaction to regulate cell proliferation and growth.[33] Inhibition of ROS production can eliminate JNK activation induced by relevant gene knockout.[34] It induces JNK phosphorylation, which result in the death of granule cells.[35] However, low levels of ROS can reduce apoptosis by activating JNK pathway or trans activating various proteins that can up regulate autophagy.[36] Endoplasmic reticulum (ER) is now considered as the main organizer of stress response pathway in cells. Similarly, JNK has a dual role in apoptosis induced by endoplasmic reticulum stress. ERmembrane-resident proteins can activate JNK pathway under endoplasmic reticulum stress, which inhibits apoptosis in the early stage of endoplasmic reticulum stress response and promotes apoptosis in the late stage.[37] At the same time, calpain protein is involved in the activation of ASK1 and JNK response pathway, which plays a role in the apoptosis response induced by ER stress.[38]

In addition to the exposure to ROS and other factors, environmental changes such as ultraviolet, high temperature and hypertonic may also cause adaptive responses to stress. For example, JNK activation selectively regulates UV mediated apoptosis. Upstream kinase phosphorylated JNK can enhance the UV induced apoptosis response of fibroblasts.[39] The activation of $\mathrm{JNK}$ is also directly related to the apoptosis induced by $\gamma$ - radiation.[40]

\subsubsection{JNK with autophagy and apoptosis}

Many studies suggest that death of neurons in hippocampus is one of the mechanisms of depression. And JNK-MAPK pathway plays a key role in regulating autophagy and apoptosis balance.[41] The study found that JNK has the kinase dependent and independent functions in the process of tumor necrosis factor (TNF) and toll-like receptor (TLR)activating necroptotic cell death.[42] As for the process of TNF driving cell death, JNK has a dual action too. The early and transient activation of JNK is a signal to promote cell survival[43], while the long-term and sustained activation of JNK can inhibit NF-KB mediated gene expression and lead to cell death.[44] The loss of neurons caused by apoptosis and autophagy is likely to be involved in the damage of stress to hippocampus, resulting in depression. There are many effective factors in this process.
Forkhead transcription factor (FOXO) is a key effector of JNK mediated autophagy and apoptosis.[45] It is considered that JNK is involved in the negative regulation of neuron FOXO-dependent autophagy.[46] Its main role is FOXO nuclear localization and to increase the expression of genes related to oxidative stress tolerance.[47] This regulatory process also depends on AP-1 transcriptional activity.[48] In the apoptotic response, the regulation of $\mathrm{JNK}$ on $\mathrm{FOXO}$ activity depends on $\operatorname{Bim}, \operatorname{Bad}[49]$ and other proteins.

Another key factor to activate JNK signal is CTRP3. When CTRP3 gene is knocked down, JNK signaling pathway activated by LPS in microglia is blocked. Simultaneously, hippocampal neuron death and apoptosis are inhibited and depressive-like behavior is alleviated markedly.[22]Beyond that, Bcl-2-associated $\mathrm{X}$ protein (Bax)[50] and other proteins are also involved in JNK mediated apoptosis. Moreover, JNK1 can mediate the phosphorylation of Bcl-2, destroying Bcl-2 / Beclin 1 complex to stimulate starvation induces autophagy.[51]

\subsection{JNK and brain development}

As is known, JNK has many physiological functions in the process of brain development. First, JNK1 is highly active in the developing cortex. It was found that JNK1 could not only regulate cell migration in the brain by regulating the rate of movement from multipolar phase to neuron during cortical development[52], but also mediate the migration of cortical interneurons to the developing cerebral cortex.[53] In addition, JNK can change the metaplasia characteristics of dentate granule cells. The increase of JNK level can enhance the brain's metaplasticity, which refers to the induction of long-term potentiation (LTP) in the hippocampus regulated by the expression of long-term depression (LTD) at the same synapse.[54] The study of JIP1-JNK signal axis also found that JNK activation mediated by JIP1 regulates hippocampal dependence, synaptic plasticity and memory.[55] The changes of these physiological processes are closely related to the onset of depression.

\subsubsection{JNK and neurogenesis}

The hippocampus, especially the ventral hippocampus, is involved in anxiety and depression.[56] Studies have shown that the volume of hippocampus in depression patients will be reduced.[57] Atrophy and loss of neurons and glial cells is one of the causes of depression. Regional brain volume is not only involved in the occurrence of depression, but also may be related to the clinical response rate and degree of antidepressants.[58] Normal neurogenesis is an important part of the body's adaptive response, which can buffer the main stress system.[59] Decreased neurogenesis is associated with anxiety and depression. Electroconvulsive sezures (ECS) can improve many anxiety and depression like behaviors, and complete hippocampal neurogenesis is a necessary condition for ECS to play a role.[60] It is found that neurogenesis is related to HPA axis disorder and has a direct role in depression.[61] 
Hippocampal neurogenesis is regulated by JNK.[62] This activity plays an important role in regulating emotion in the newborn hippocampal granule cells.[63] It was found that mice lacking JNK inhibitors exhibited increased neurogenesis in the adult hippocampus, including increased cell proliferation and increased ventral regional maturity. There are also research findings that inhibition of JNK in hippocampal niches increases the number of granule cells and reduces anxiety and depression behavior.[64] Therefore, JNK can be used as the target of antidepressant therapy. Ideal new drugs can supplement new neurons by regulating JNK expression, and then help to restore the stress system and improve depression like state.[65]

\subsubsection{JNK and synaptic plasticity}

Abnormal dendrite structure may lead to neurodevelopmental abnormality and mental illness in the nervous system. In the fine regulation of dendrite shape and synaptic connections, JNK plays an important role.[66] The activation of $\mathrm{JNK}$ is a necessary factor for axon formation. It was found that JNK was highly enriched in axons. At the same time, inhibition of JNK expression can prevent axon formation.[67] The interruption of JNK1 signal leads to the disorder of dendrite complex, including the decrease of dendrite branches and morphological changes.[68]

Different forms of synaptic plasticity, including longterm depression (LTD) and long-term potentiation (LTP), can affect the normal function of the brain by changing the state of synaptic function. The change of JNK activity is considered to be the key factor of synaptic plasticity in depression pathogenesis. Proteins of Ras family are the molecular switches of JNK and other signal transduction cascade, which can control synaptic plasticity and cause depression and other mental diseases.[69] For example, Rap signal can specifically control many kinds of synaptic plasticity through bulk membrane JNK.[70] Specifically, this process can achieve synaptic clearance in the terminal synaptic pool by inducing the long cytoplasmic AMPA receptor subunit.[71] The control of JNK signal may not only enhance synaptic inhibition, but also indirectly promote LTP through the damage of inhibition.[72] It is found that NMDA receptor is a key site of synaptic plasticity. In the experiment, NMDA signal was not converged with other chronic neurotransmitters in JNK pathway, and then short-term synaptic inhibition was induced.[73]

In addition to the postsynaptic mechanism of $\mathrm{JNK}$, there are also studies on the presynaptic location of JNK and its physiological role in this area. Presynaptic components contain a large number of JNK proteins and their activation forms, which play their own roles in the normal function of synapses. For example, JNK has been found to regulate presynaptic vesicles to release TSNARE protein, which may play an important role in the changes of synaptic plasticity.[74]

\subsection{JNK and other physiological processes}

In addition to the above physiological processes, JNK may also participate in a variety of other physiological activities related to the onset of depression, including gene expression changes, cytoskeleton changes and intracellular transport activities, cell cycle changes, etc.

\subsubsection{JNK and gene expression}

The pathogenesis of depression involves many changes in gene expression. The most typical substrate of JNK is various nuclear transcription factors, including Jun, Atf2, Myc, ELK1, etc. By phosphorylating these transcription factors, JNK can regulate the expression of many genes. Among them, there are many gene expression products that may be related to the pathogenesis of depression. As the most important substrate of JNK, c-jun can activate many proteins through transcriptional activation and post-translational modification, for example, p73, which is of great significance in apoptosis. The expression of c-jun increases the half-life of p73 by inhibiting proteasome mediated degradation, thus enhancing the transcriptional activity of p73.[75] In addition, JNK signal transduction pathway plays a role in the transcription response mediated by activating transcription factor-2 (ATF2). Concretely, JNK can phosphorylates ATF2 on two closely spaced threonine residues in the terminal activation region of $\mathrm{NH} 2 .[76]$ In addition to transcriptional activation, JNK can also participate in the mRNA splicing process through phosphorylation and regulate the expression of final products. The splicing factor 45 (SPF45) is an example in which JNK is involved in changing the splicing site, expression level and function.[77] The activation of JNK causes the change of gene expression, which may be the key to relationship between depression and JNK signal.

\subsubsection{JNK and cytoskeleton}

The occurrence of depression and the effect of antidepressant treatment may be related to the change of cytoskeleton protein. Microtubule stability has influence on behavior and antidepressant effect of rats. In this process, JNK may play a regulatory role. By activating downstream substrates, JNK can be involved in the regulation of cytoskeleton and intracellular transporters. Specifically, JNK participated in cytoskeleton regulation depends on substrate proteins such as microtubule associated protein and actin associated protein. WD40repeat protein 62 (WDR62) is a spindle polar protein necessary for normal cell division and differentiation during brain development. Phosphorylation of WDR62 mediated by JNK can negatively regulates microtubule binding.[78] Belonging to actin family, SMTNL2 plays a role in the process of myoblast to myotube transformation. The phosphorylation of JNK can regulate the expression of SMTNL2.[79] Furthermore, JNK phosphorylation pathway can also mediate vesicle transport. Many vesicles associated proteins are regulated by JNK. In conclusion, there is a close relationship 
between the change of cytoskeleton and the occurrence of depression and antidepressant treatment, but the role of JNK in it needs to be further elucidated.

\subsubsection{JNK and cell cycle}

The cell cycle of hippocampal nerve cells was influenced by electroshock and many antidepressants. At the same time, JNK plays a part in the process of cell cycle. C-Jun is favorable for cells to enter cell cycle smoothly, while cells lacking c-Jun will experience long-term cell cycle arrest.[80] Transcription factors like AP-1 regulated by JNK have positive functions in cell proliferation.[81] Study of in situ Hi-C maps of DNA loops has shown that the composition of AP-1 transcription factor complexes possiblely contribute to reconnection of chromatin interactions in cell type and tissue-specific manners.[82] It is reasonable to speculate that JNK regulates the cell cycle of neurons, which is one of the mechanisms that JNK participates in the pathogenesis of depression.

Table. 1. Regulation of JNK activity in depression.

\begin{tabular}{|c|c|c|c|}
\hline Stress & JNK activity & Participation factors & Effect \\
\hline LPS, etc & $\uparrow$ & proinflammatory factors and GR,etc & $\begin{array}{l}\text { inflammatory response } \\
\text { insulin resistance } \\
\text { endocrine disorder }\end{array}$ \\
\hline ROS,UV,etc & $\uparrow$ & calpain protein,etc & $\begin{array}{c}\text { ER stress } \\
\text { cell apoptosis }\end{array}$ \\
\hline TNF,TLR activation & $\uparrow$ & NF-KB,FOXO,etc & Autophagy, apoptosis \\
\hline Multiple stimuli & $\uparrow$ & $\begin{array}{c}\text { granule cells and } \\
\text { neurons } \\
\text { in hippocampus }\end{array}$ & $\begin{array}{c}\text { Neurogenesis decrease } \\
\text { Neuron loss }\end{array}$ \\
\hline LTD and LTP & $\uparrow$ & Rap,NMDA,etc & synaptic plasticity change \\
\hline Multiple stimuli & $\uparrow$ & c-Jun,Atf2,ELK and Myc,etc & Gene expression change \\
\hline Multiple stimuli & $\uparrow$ & WDR62,SMTNL2,etc & cytoskeleton change \\
\hline Drugs and electroshock & & c-Jun,AP-1,etc & cell cycle arrest \\
\hline
\end{tabular}

\section{$4 \mathrm{JNK}$ and treatment of depression}

The combination of JNK and downstream factors has many important regulatory functions[83]. It implies that JNK has great potential as a drug target of clinical treatment. It has been found that JNK can be used as an effective treatment target for many diseases, including neurodegenerative diseases, immune diseases, cancer, metabolic diseases, etc.[84] In the pathogenesis of depression, JNK pathway may be involved in many physiological processes, such as inflammation, cell proliferation, apoptosis, neurogenesis and protein expression. Therefore, inhibition of JNK can be used as a new therapeutic target for emotional disorders.

At present, the research on JNK targeted therapy is still at the level of animal experiments, with mouse as a commonly used animal model. Due to the differences between human and mouse tissues, the application of research results is limited. Most of JNK targeted drugs effective in animal experiments are JNK inhibitors. Most of them belong to non-specific JNK inhibitors, while a few studies also focus on subtype specific JNK inhibitors. [85] Since different subtypes of JNK have different distribution and specific functions, the research and development of subtype specific JNK inhibitors has great potential in the future.

\section{Conclusion and prospect}

The pathogenesis of depression has always been drawing people's attention. At present, although there are more and more researches on how MAPK signaling pathway get involved in depression and other mental diseases, the specific mechanism of how JNK signaling pathway affects depression is still not clear. In current studies, $\mathrm{JNK}$ is involved in the pathogenesis of depression through various processes such as neuroinflammation, oxidative stress, cell death and neurogenesis. However, the research is mostly at the level of cell or animal model, and the research focus is relatively unitary, lacking integration and systematization. We need to do more research to better understand the role of JNK in the pathogenesis of depression, so as to develop new targeted antidepressants. In addition, many problems remain unsolved, such as why similar abnormal JNK mechanisms lead to different clinical manifestations and at which stage of depression the abnormal expression of JNK makes an impact.

\section{Acknowledgment}

At first, I would like express my gratitude to Dr.Tao Heqing, who has provided me with valuable guidance in every stage of the writing.

My sincere thanks are also given to Dr.Hu Qihang for his professional instructions.

Besides, I am extremely grateful for Mrs.Liu qin for her selfless assistance throughout the revision of the article.

Last but not least, I would like to thank my family and friends, for their support and encouragement.

\section{References}

1. L. Sjoberg, B. Karlsson, A.R. Atti, et al. J Affect Disord. Prevalence of depression: Comparisons of different depression definitions in population-based samples of older adults, 221, 123-131(2017) 
2. C. Menard, G.E. Hodes, S.J. Russo, Neuroscience. Pathogenesis of depression: Insights from human and rodent studies, 321, 138-162(2016)

3. L. Chang, M. Karin, Nature. Mammalian MAP kinase signalling cascades, 410(6824), 37-40(2001)

4. M. Guha, N. Mackman, Cell Signal. LPS induction of gene expression in human monocytes, 13(2),85-94 (2001)

5. D.T. Ho, A.J. Bardwell, M. Abdollahi, et al. J Biol Chem.A docking site in MKK4 mediates high affinity binding to JNK MAPKs and competes with similar docking sites in JNK substrates, 278(35), 3266272(2003)

6. A.E. Matitau, T.V. Gabor, R.M. Gill, et al. J Biol Chem, MEKK2 kinase association with 14-3-3 protein regulates activation of c-Jun $N$-terminal kinase, 288(39) ,28293-302(2013)

7. E. Alvarez, I.C. Northwood, F.A. Gonzalez, et al. J Biol Chem Pro-Leu-Ser/Thr-Pro is a consensus primary sequence for substrate protein phosphorylation. Characterization of the phosphorylation of c-myc and c-jun proteins by an epidermal growth factor receptor threonine 669 protein kinase, 266(23), 15277-85(1991)

8. S. Gupta, T. Barrett, A.J. Whitmarsh, et al. EMBO J. Selective interaction of JNK protein kinase isoforms with transcription factors, 15(11) ,2760-70(1996)

9. T.C. Whisenant, D.T. Ho, R.W. Benz, et al. PLoS Comput Biol. Computational prediction and experimental verification of new MAP kinase docking sites and substrates including Gli transcription factors, 6(8) (2010).

10. J.P. Li, Y.N. Fu, Y.R. Chen, et al. J Biol Chem. JNK pathway-associated phosphatase dephosphorylates focal adhesion kinase and suppresses cell migration, 285(8),5472-8(2010)

11. C.Y. Yang, J.P. Li, L.L. Chiu, et al. J Immunol. Dual-specificity phosphatase 14 (DUSP14/MKP6) negatively regulates TCR signaling by inhibiting TAB1 activation, 192(4) ,1547-57(2014)

12. M.M. Fu, E.L. Holzbaur, J Cell Biol. JIP1 regulates the directionality of APP axonal transport by coordinating kinesin and dynein motors, 202(3) ,495-508 (2013)

13. T. Satake, K. Otsuki, Y. Banba, et al. BMC Cell Biol. The interaction of Kinesin-1 with its adaptor protein JIP1 can be regulated via proteins binding to the JIP1-PTB domain, 14 ,12(2013)

14. K.A. Schachter, Y. Du, A. Lin, et al. J Biol Chem. Dynamic positive feedback phosphorylation of mixed lineage kinase 3 by JNK reversibly regulates its distribution to Triton-soluble domains, 281(28), 19134-44(2006)

15. Z. Lu, Y. Miao, I. Muhammad, et al. Chem Biol Interact. Colistin-induced autophagy and apoptosis involves the JNK-Bcl2-Bax signaling pathway and JNK-p53-ROS positive feedback loop in PC-12 cells, 277,62-73(2017)
16. X. Chen, C. Liu, R. Zhao, et al. Mol Pharm. Synergetic and Antagonistic Molecular Effects Mediated by the Feedback Loop of p53 and JNK between Saikosaponin D and SP600125 on Lung Cancer A549 Cells, 15(11),4974-4984(2018)

17. S. Wang, H. Li, S. Weng, et al. iScience. White Spot Syndrome Virus Establishes a Novel IE1/JNK/c-Jun Positive Feedback Loop to Drive Replication, 23(1), 100752(2019)

18. M. Solas, G. Gerenu, F.J. Gil-Bea, et al. J Neuroendocrinol. Mineralocorticoid receptor activation induces insulin resistance through c-Jun $N$-terminal kinases in response to chronic corticosterone: cognitive implications, 25(4),350-6 (2013)

19. J. Zhang, W. Lin, M. Tang, et al. Psychoneuroendocrinology. Inhibition of JNK ameliorates depressive-like behaviors and reduces the activation of pro-inflammatory cytokines and the phosphorylation of glucocorticoid receptors at serine 246 induced by neuroinflammation, 113,104580(2019)

20. G. Sabio, R.J. Davis, Semin Immunol. TNF and MAP kinase signalling pathways, 26(3), 23745(2014)

21. B. Zhang, M. Li, L. Chen, et al. Cell Res. The TAK1-JNK cascade is required for IRF3 function in the innate immune response, 19(4),(2009) 41228(2009)

22. J. Meng, D.M. Wang, L.L. Luo, Biomed Pharmacother. CTRP3 acts as a novel regulator in depressive-like behavior associated inflammation and apoptosis by meditating p38 and JNK MAPK signaling, 120, 109489 (2019)

23. M. Spiliotaki, V. Salpeas, P. Malitas, et al. Psychoneuroendocrinology. Altered glucocorticoid receptor signaling cascade in lymphocytes of bipolar disorder patients, 31(6), 748-60(2006)

24. T.R. Bomfim, L. Forny-Germano, L.B. Sathler, et al. $\mathrm{J}$ Clin Invest. An anti-diabetes agent protects the mouse brain from defective insulin signaling caused by Alzheimer's disease- associated Abeta oligomers, 122(4), 1339-53(2012)

25. M.S. Han, D.Y. Jung, C. Morel, et al. Science. JNK expression by macrophages promotes obesityinduced insulin resistance and inflammation, 339(6116), 218-22(2013)

26. J. Lanuza-Masdeu, M.I. Arevalo, C. Vila, et al. Diabetes. In vivo JNK activation in pancreatic betacells leads to glucose intolerance caused by insulin resistance in pancreas, 62(7), 2308-17(2013)

27. A. Ammendrup, A. Maillard, K. Nielsen, et al. Diabetes. The c-Jun amino-terminal kinase pathway is preferentially activated by interleukin-1 and controls apoptosis in differentiating pancreatic betacells, 49(9), 1468-76(2000)

28. E.T. Uchoa, P.B. Marangon, R. Rorato, et al. J Endocrinol. Adrenalectomy impairs insulin-induced 
hypophagia and related hypothalamic changes, 242(2), 125-138 (2019)

29. S. Vernia, J. Cavanagh-Kyros, T. Barrett, et al. Genes Dev. Diet-induced obesity mediated by the JNK/DIO2 signal transduction pathway, 27(21), 2345-55(2013)

30. J.M. Kyriakis, P. Banerjee, E. Nikolakaki, et al. Nature. The stress-activated protein kinase subfamily of c-Jun kinases, 369(6476), 156-60(1994)

31. N. Galeotti, C. Ghelardini, Int J Neuropsychopharmacol. Regionally selective activation and differential regulation of ERK, JNK and p38 MAP kinase signalling pathway by protein kinase $C$ in mood modulation, 15(6), 781-93 (2012)

32. S.G. Rosa, A.P. Pesarico, C.W. Nogueira. Prog Neuropsychopharmacol Biol Psychiatry. mTrifluoromethyl-diphenyl diselenide promotes resilience to social avoidance induced by social defeat stress in mice: Contribution of opioid receptors and MAPKs, 82, 123-135(2018)

33. A.G. Toshniwal, S. Gupta, L. Mandal, et al. Dev Cell. ROS Inhibits Cell Growth by Regulating 4EBP and S6K, Independent of TOR, during Development, 49(3), 473-489 (2019)

34. J. Sun, P. Li, J. Yang. Biochem Biophys Res Commun. Repressing of NHERF1 inhibits liver cancer progression by promoting the production of ROS, 509(1), 8-15(2019)

35. H. Yang, Y. Xie, D. Yang, et al. Oncotarget. Oxidative stress-induced apoptosis in granulosa cells involves JNK, p53 and Puma, 8(15), 2531025322(2017)

36. V.O. Kaminskyy, B. Zhivotovsky. Antioxid Redox Signal. Free radicals in cross talk between autophagy and apoptosis, 21(1), 86-102(2014)

37. M. Brown, N. Strudwick, M. Suwara, et al. J Cell Sci. An initial phase of JNK activation inhibits cell death early in the endoplasmic reticulum stress response, 129(12), 2317-2328(2016)

38. Y. Tan, N. Dourdin, C. Wu, et al. J Biol Chem. Ubiquitous calpains promote caspase-12 and JNK activation during endoplasmic reticulum stressinduced apoptosis, 281(23), 16016-24(2006)

39. N.L. Johnson, A.M. Gardner, K.M. Diener, et al. J Biol Chem. Signal transduction pathways regulated by mitogen-activated/extracellular response kinase kinase kinase induce cell death, 271(6), 322937(1996)

40. Y.R. Chen, X. Wang, D. Templeton, et al. J Biol Chem. The role of $c$-Jun $N$-terminal kinase (JNK) in apoptosis induced by ultraviolet $C$ and gamma radiation. Duration of JNK activation may determine cell death and proliferation, 271(50), 31929-36(1996)

41. X. Sui, N. Kong, L. Ye, et al. Cancer Lett. p38 and JNK MAPK pathways control the balance of apoptosis and autophagy in response to chemotherapeutic agents, 344(2), 174-9(2014)
42. M. Cao, F. Chen, N. Xie, et al. Cell Death Dis. c-Jun $N$-terminal kinases differentially regulate TNF-and TLRS-mediated necroptosis through their kinasedependent and -independent activities, 9(12), 1140(2018)

43. A. Roulston, C. Reinhard, P. Amiri, et al. J Biol Chem. Early activation of c-Jun N-terminal kinase and 38 kinase regulate cell survival in response to tumor necrosis factor alpha, 273(17), 10232-9(1998)

44. J.L. Luo, H. Kamata, M. Karin, J Clin Invest. IKK/NF-kappaB signaling: balancing life and death--a new approach to cancer therapy, 115(10), 2625-32(2005)

45. W. Bi, L. Xiao, Y. Jia, et al. J Biol Chem. c-Jun Nterminal kinase enhances MST1-mediated proapoptotic signaling through phosphorylation at serine 82, 285(9), 6259-64(2010)

46. P. Xu, M. Das, J. Reilly, et al. Genes Dev. JNK regulates FoxO-dependent autophagy in neurons, 25(4), 310-22(2011)

47. M.C. Wang, D. Bohmann, H. Jasper. Cell. JNK extends life span and limits growth by antagonizing cellular and organism-wide responses to insulin signaling, 121(1), 115-25(2005)

48. M. Uno, S. Honjoh, M. Matsuda, et al. Cell Rep. $A$ fasting-rTimes New RomanTimes New Romanesponsive signaling pathway that extends life span in C. elegans, 3(1), 79-91(2013)

49. J.M. Ness, C.A. Harvey, A. Strasser, et al. Brain Res. Selective involvement of BH3-only Bcl-2 family members Bim and Bad in neonatal hypoxia-ischemia, 1099(1), 150-9(2006)

50. S. Zhou, Y. Wang, J.J. Zhu. ACS Appl Mater Interfaces. Simultaneous Detection of Tumor Cell Apoptosis Regulators Bcl-2 and Bax through a Dual-Signal-Marked Electrochemical Immunosensor, 8(12), 7674-82(2016)

51. Y. Wei, S. Pattingre, S. Sinha, et al. Mol Cell. JNK1mediated phosphorylation of Bcl-2 regulates starvation-induced autophagy, 30(6), 678-88(2008)

52. N. Westerlund, J. Zdrojewska, A. Padzik, et al. Nat Neurosci. Phosphorylation of SCG10/stathmin-2 determines multipolar stage exit and neuronal migration rate, 14(3), 305-13(2011)

53. A.K. Myers, D.W. Meechan, D.R. Adney, et al. J Neurosci. Cortical interneurons require Jnk1 to enter and navigate the developing cerebral cortex, 34(23), 7787-801(2014)

54. M. Yousef, E. Babur, S. Delibas, et al. J Mol Neurosci. Adult-Onset Hypothyroidism Alters the Metaplastic Properties of Dentate Granule Cells by Decreasing Akt Phosphorylation, 68(4), 647657(2019)

55. C. Morel, T. Sherrin, N.J. Kennedy, et al. J Neurosci. JIP1-Mediated JNK Activation Negatively Regulates Synaptic Plasticity and Spatial Memory, 38(15), 3708-3728(2018)

56. N. Padilla-Coreano, S.S. Bolkan, G.M. Pierce, et al. 
Neuron. Direct Ventral Hippocampal-Prefrontal Input Is Required for Anxiety-Related Neural Activity and Behavior, 89(4), 857-66(2016)

57. S. MacMillan, P.R. Szeszko, G.J. Moore, et al. J Child Adolesc Psychopharmacol. Increased amygdala: hippocampal volume ratios associated with severity of anxiety in pediatric major depression, 13(1), 65-73(2003)

58. G.M. MacQueen, K. Yucel, V.H. Taylor, et al. Biol Psychiatry. Posterior hippocampal volumes are associated with remission rates in patients with major depressive disorder, 64(10), 880-3(2008)

59. A. Tanti, C. Belzung. Neuroscience. Neurogenesis along the septo-temporal axis of the hippocampus: are depression and the action of antidepressants region-specific? 252, 234-52(2013)

60. R.J. Schloesser, S. Orvoen, D.V. Jimenez, et al. Brain Stimul. Antidepressant-like Effects of Electroconvulsive Seizures Require Adult Neurogenesis in a Neuroendocrine Model of Depression, 8(5), 862-7(2015)

61. J.S. Snyder, A. Soumier, M. Brewer, et al. Nature. Adult hippocampal neurogenesis buffers stress responses and depressive behaviour, 476(7361), 458-61(2011)

62. R.D. Castro-Torres, J. Landa, M. Rabaza, et al. Mol Neurobiol. JNK Isoforms Are Involved in the Control of Adult Hippocampal Neurogenesis in Mice, Both in Physiological Conditions and in an Experimental Model of Temporal Lobe Epilepsy, 56(8), 5856-5865(2019)

63. P. Hollos, F. Marchisella, E.T. Coffey. Brain Plast. JNK Regulation of Depression and Anxiety, 3(2), 145-155(2018)

64. H. Mohammad, F. Marchisella, S. Ortega-Martinez, et al. Mol Psychiatry. JNK1 controls adult hippocampal neurogenesis and imposes cellautonomous control of anxiety behaviour from the neurogenic niche, 23(2), 487(2018)

65. A. Surget, A. Tanti, E.D. Leonardo, et al. Mol Psychiatry. Antidepressants recruit new neurons to improve stress response regulation, 16(12), 117788(2011)

66. B. Bjorkblom, N. Ostman, V. Hongisto, et al. J Neurosci. Constitutively active cytoplasmic c-Jun Nterminal kinase 1 is a dominant regulator of dendritic architecture: role of microtubuleassociated protein 2 as an effector, 25(27), 635061(2005)

67. A.A. Oliva, Jr., C.M. Atkins, L. Copenagle, et al. J Neurosci. Activated c-Jun N-terminal kinase is required for axon formation, 26(37), 9462-70(2006)

68. E. Komulainen, J. Zdrojewska, E. Freemantle, et al. Front Cell Neurosci. JNK1 controls dendritic field size in L2/3 and L5 of the motor cortex, constrains soma size, and influences fine motor coordination, $\mathbf{8}$, 272(2014)

69. R.L. Stornetta, J.J. Zhu. Neuroscientist. Ras and Rap signaling in synaptic plasticity and mental disorders, 17(1), 54-78(2011)

70. L. Zhang, P. Zhang, G. Wang, et al. Neuron. Ras and Rap Signal Bidirectional Synaptic Plasticity via Distinct Subcellular Microdomains, 98(4), 783800(2018)

71. A. Kielland, G. Bochorishvili, J. Corson, et al. Neuron. Activity patterns govern synapse-specific AMPA receptor trafficking between deliverable and synaptic pools, 62(1), 84-101(2009)

72. H. Yang, M.J. Courtney, P. Martinsson, et al. Eur J Neurosci. Hippocampal long-term depression is enhanced, depotentiation is inhibited and long-term potentiation is unaffected by the application of a selective c-Jun N-terminal kinase inhibitor to freely behaving rats, 33(9), 1647-55 (2011)

73. H. Kaphzan, K.J. O'Riordan, K.P. Mangan, et al. PLoS One. NMDA and dopamine converge on the NMDA-receptor to induce ERK activation and synaptic depression in mature hippocampus, 1, 138 (2006)

74. S. Biggi, L. Buccarello, A. Sclip, et al. Neural Plast. Evidence of Presynaptic Localization and Function of the c-Jun N-Terminal Kinase, 2017, 6468356 (2017)

75. W.H. Toh, M.M. Siddique, L. Boominathan, et al. J Biol Chem. c-Jun regulates the stability and activity of the p53 homologue, p73, 279(43), 44713-22 (2004)

76. S. Gupta, D. Campbell, B. Derijard, et al. Science. Transcription factor ATF2 regulation by the JNK signal transduction pathway, 267(5196), 389-93 (1995)

77. A.M. Al-Ayoubi, H. Zheng, Y. Liu, et al. Mol Cell Biol. Mitogen-activated protein kinase phosphorylation of splicing factor 45 (SPF45) regulates SPF45 alternative splicing site utilization, proliferation, and cell adhesion, 32(14), 288093(2012)

78. N.R. Lim, Y.Y. Yeap, T.T. Zhao, et al. J Cell Sci. Opposing roles for JNK and Aurora A in regulating the association of WDR62 with spindle microtubules, 128(3), 527-40(2015)

79. E.A. Gordon, T.C. Whisenant, M. Zeller, et al. Cell Signal. Combining docking site and phosphosite predictions to find new substrates: identification of smoothelin-like-2 (SMTNL2) as a c-Jun N-terminal kinase (JNK) substrate, 25(12), 2518-29(2013)

80. E. Shaulian, M. Schreiber, F. Piu, et al. Cell. The mammalian UV response: c-Jun induction is required for exit from p53-imposed growth arrest, 103(6), 897-907(2000)

81. M. Karin, E. Shaulian. IUBMB Life. AP-1: linking hydrogen peroxide and oxidative stress to the control of cell proliferation and death, 52(1-2), 1724(2001)

82. D.H. Phanstiel, K. Van Bortle, D. Spacek, et al. Mol Cell. Static and Dynamic DNA Loops form AP-1- 
Bound Activation Hubs during Macrophage Development, 67(6), 1037-1048(2017)

83. M. Hibi, A. Lin, T. Smeal, et al. Genes Dev. Identification of an oncoprotein- and UV-responsive protein kinase that binds and potentiates the c-Jun activation domain, 7(11), 2135-48(1993)

84. A. Kumar, U.K. Singh, S.G. Kini, et al. Future Med Chem. JNK pathway signaling: a novel and smarter therapeutic targets for various biological diseases, 7(15), 2065-86 (2015)

85. M. Gehringer, F. Muth, P. Koch, et al. Expert Opin Ther Pat. c-Jun N-terminal kinase inhibitors: a patent review (2010 - 2014), 25(8), 849-72 (2015) 